\title{
Ceramic artifacts debris classification Based on fusion of physical and cultural characteristics
}

\author{
Kegang Wang ${ }^{1, a}$, Guohua Geng ${ }^{1, b}$ and Liying $\mathrm{Qi}^{2, \mathrm{c}}$ \\ 1 School of Information Science and Technology, Northwest University, Xi'an, 710127, China; \\ 2 Department of Electronic \& Information Engineering, Ankang University, Ankang, 725000, China. \\ akuwkg@aku.edu.cn, 313057508@qq.com
}

Keywords: Ceramic; Classification; Fusion; physical; cultural

\begin{abstract}
This paper presents methods of feature description and extraction of ceramic artifacts, and then use canonical correlation analysis techniques and methods for establishing the correlation between the multi-feature information criterion-function theories, to solve the case of high-dimensional small sample typical projection vector set problem. The proposed methods and performs much better than the previous methods, has received the good effect in the ceramic fragments classification experiment.
\end{abstract}

\section{Introduction}

Ceramic artifacts have the mark on the history of Chinese culture, relics artifacts damaged majority. This paper study extract methods of Ceramic artifacts physical and cultural characteristics, according to the digital information to achieve clustering analysis and synthesis of ceramic pieces feature classification for computer-aided repair damaged artifacts to provide support. (1) proposed ceramic artifacts visual information describe and Feature Extraction Method; (2) Studying of ceramic artifacts of collaborative clustering techniques based on features of glaze, decoration, shape, texture and artistic style, to achieve the same utensils ceramic artifacts fragmentation vest divided. (3) Studying the multi-step connection refinement and semi-supervised popular alignment method, and the consolidated instance migration constraints and characteristics associated classification methods to construct physical objects of pottery shards classification method. Research results can be applied to computer-aided repair broken ceramic artifacts, and to support cultural elements of ancient ceramic extraction, art style research and improve the efficiency of ceramic heritage.

\section{Characteristics of ceramic artifacts}

Styling and craftsmanship of ancient ceramics was carrying a variety of social and cultural information, it's the "living fossil" of ancient human life. Ancient ceramics with valuable archaeological value extremely, there are two levels Characteristics of ceramic artifacts are: physical attributes characteristic and cultural identity.

\subsection{Physical Characteristics}

Ceramic firing is a very complex process. The production of ceramic materials in a variety of substances and their constituents how much fuel used in firing, firing process and the firing time and many other factors are affecting the final appearance of the ceramic and ceramic-quality visual form factors, complex production process has also led to the ceramic itself is varied, more importantly, the formation of the physical characteristics of the ceramic itself, two levels of different levels:

The first is ceramic glaze and decorations are different. Due to human production of different purposes, use of materials, processing handmade painted pattern that none of the same, this feature is the difference between individual ceramic bases. The second is ceramic microscopic images are different. In microscopic images, we can see the ceramic crystal structure, bubbles, cracks and other 
opening film. This structure is a fired material, affect the formation of the firing process, not man-intentional.

\subsection{Cultural characteristics}

Different times and different types of ceramic have different culture styles. Ceramic glaze mix, composition design, decoration and styling and the overall shape ceramics, etc. This series consists of colors, patterns, lines and other physical attributes triggered by visual ornamentation beauty features, Beautiful characteristic constitutes an important element of the cultural identity of the ceramic, giving different style art enjoyment.

\section{Feature description and extraction of ceramic artifact}

\subsection{Ornamentation information representation and feature extraction methods}

Ornamentation is an important feature for the visual appearance of ceramic artifacts, and its important factor in ceramic discriminator and category for pottery shards. Existing a variety of shapes characteristic methods for digital images[1-3], for example: the same moment, the edge histogram, Fourier descriptors, shape parameter for quantitative measurement method, etc, these methods have the lack of perfect mathematical model is only suitable for describing the target of local nature, as reflected in target shape similarity of feature space with the human visual system is perceived similarities are quite different. We use multi-resolution image processing Ceramic image, according to the transform modulus maxima obtained ceramic ornamentation multi-scale to compute invariant moment's physical characteristics of the border, and with ornamentation constitutes a semantic network of high-level semantic features semantic decoration, ornamentation with simultaneous partial morphological description with the overall nature of the capabilities and fully characterize ceramic ornamentation cultural information.

\subsection{Glaze information expression and feature extraction method}

Porcelain glaze is important information, are currently using some of the modification of the terms described on the porcelain glaze. Glaze ceramic glaze descriptive words reflects the rich classes and unique cultural characteristics of Chinese ceramics color level of detail, but the main basis for the human eye to glaze the feeling of depth and shading to distinguish, with greater subjectivity, can not be accurately calculated and expression. Fist, we use the uniform color space, spectral reflectance spectrum curve method for quantitative characterization of glaze to calculate the histogram, central moments, frequency domain coefficient to represent the color physical characteristics, secondly, we obtain a semantic networks base on combined physical characteristics and which relevant glaze vocabulary for categories, dimensionality reduction glaze semantic feature vector generation at last. The method above can reduction glaze information distortion, and low complexity, consistent with human visual perception, fully reflect the glaze porcelain culture on the understanding and definition of different types of expression of the intrinsic characteristics of porcelain glaze.

\subsection{Times feature expression and extraction method}

There is bubbles, weathered spots structure, surface morphology contained in Ceramic glazed microscopic image, which feature reflects the tiny cracks and other characteristics of the era ceramics, which is the important common feature for same category Ceramics. Microscopic images of ancient ceramic pre-processing and segmentation, separation of the crystalline phase microstructure contour map, and stereological methods combined with microscopic image measurement and analysis [4], access universal parameters for the ceramic matrix components and the relationship between the appearances of texture to provide a basis for the further fragmentation accurate classification.

\subsection{Multi-feature fusion}

We had the same pattern optimized combination of different feature vectors, which can take advantage of a variety of features to interpret joint understanding on the ceramic. Existing serial and parallel fusion methods are carried out in the high dimensional space to extract integration feature, such an approach does not have the significant capacity to eliminate significant correlation amongst the initial characteristic. We use canonical correlation analysis techniques and method for 
establishing the correlation between the multi-feature information criterion function theories, to solve the case of high-dimensional small sample typical projection vector set problem. The above methods through canonical correlation feature combination of information extraction to obtain ceramic fragments fused feature, effective use of the characteristics of the link between information and eliminates redundant information to improve the debris classification accuracy.

\section{Clustering and migration based on a collaborative learning ceramic shards classification}

First, the use of collaborative clustering ideas to achieve the same artifacts ceramic artifacts fragmentation vest division. Texture, glaze, decoration and crystal structure of micro-images, bubbles, cracks and other opening film is characterized by a strong distinction between the individual ceramic artifacts basis, and different ceramic fragments which belonging to the same artifacts with two levels of physical and cultural common feature.

Secondly, ceramic fragments clustering results subset elements constitute the "pieces" to recover artifacts library. Traditional machine learning method of fragment classification need a lot of manpower to mark training data calibration [5], apply only to the training data and the classification of data with the same distribution. We use the automatic classification based on transfer learning annotation methods according to build a classification model objects form ceramic fragments[6-7], and the realization of ceramic fragments form a subset of the clustering results attribution classification label. During the above process, we use the instance-based transfer learning intelligent classification techniques and feature-based representation transfer learning intelligent classification techniques, according to the advantages and disadvantages of these two algorithms to merge the two methods, applied new method to the ceramic debris classification process, we received good experiment results.

\section{Conclusion}

Our method will be the physical characteristics of ceramics and cultural feature fusion, greatly enriched the digital characteristic information of ceramic artifacts, provides more effective data support for the fragment classification. The proposed method takes into account the ceramic artifacts category, different characteristics, makes full use of relevant theories on transfer learning, classification of the migration process small sample size, has received the good effect in the ceramic fragments classification experiment.

\section{Acknowledgement}

This work is supported by the National Natural Science Foundation of China (No. 631773117) and Natural Science Basic Research Plan in Shaanxi Province of China (Program No. 2014JM8358).

\section{Reference}

[1]B. John Oommen, A. Thomas, “Anti-Bayesian" parametric pattern classification using order statistics criteria for some members of the exponential family[J], Pattern Recognition, 2014,47:40-55

[2]Kyoungok Kim, Jaewook Lee, Sentiment visualization and classification via semi-supervised nonlinear dimensionality reduction[J], Pattern Recognition 47 (2014) :758-768

[3] D.Comaniciu,P.Meer. Mean Shift:A robust approach toward feature space analysis.IEEE PAMI,24:603-619,2002.

[4] J. Winn, A. Criminisi, T. Minka, Object categorization by learned universal visual dictionary.Proceedings of the Tenth IEEE International Conference on Computer Vision, vol. 2, 2005, pp. 1800-1807 
[5] Bai Xue, Luo Siwei. Combining bag of words model and information theoretic method for image clustering.Advances in Neural Network Research and Applications ,2010, 67(5),pp. 423-430.

[6] KeGang Wang,Guohua Geng.Emotional Image Retrieval with Interactive Harmony search Algorithm. Journal of Computational Information Systems,2012,8(15):6175-6183

[7] Guo, J., Guo, H. \& Wang, Z. An Activation Force-based Affinity Measure for Analyzing Complex Networks. Sci. Rep. 1, 113; DOI:10.1038/srep00113 (2011). 